\title{
Improving the polishing accuracy by determining the variance of the friction coefficient
}

\author{
A. Kelm \\ HTW Aalen, Beethovenstr. 1, 73430 Aalen, Germany \\ Andreas.Kelm@htw-aalen.de \\ R. Boerret \\ HTW Aalen, Beethovenstr. 1, 73430 Aalen, Germany \\ S. Sinzinger \\ TU Ilmenau, Postfach 100565, 98684 Ilmenau, Germany
}

The variation of the friction coefficient in the polishing process is investigated comparing the results of a numerical simulation with polishing experiments. To improve the accuracy of the predicted wear in the simulation a method is presented to measure the friction coefficient in relation to the relative velocity using an offset tool.

[DOI: http://dx.doi.org/10.2971/jeos.2012.12049]

Keywords: Polishing, CCP, simulation, Preston coefficient, friction coefficient

\section{INTRODUCTION}

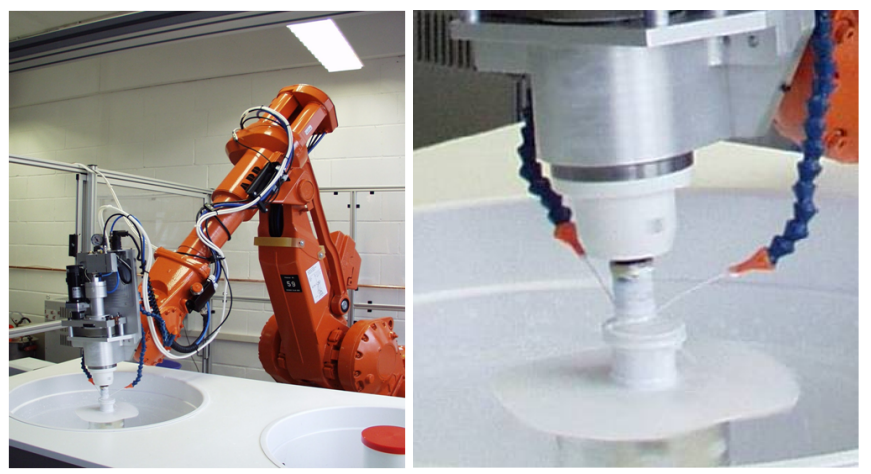

FIG. 1 left: robot used for polishing, right: polishing pad on surface with polishing slurry

A part generated by grinding, milling or turning often has a rough surface. The polishing process is used to smooth surface structures. Figure 1 shows as an example the automated polishing of a lens surface. On the left side the industrial robot colored orange is moving a polishing head. It consists of a spindle that is driven by an electric motor with feedback control. With a pressure cylinder mounted on top of the spindle a predefined force can be applied to the tool that is attached to the lower end of the spindle.

Figure 1, right side shows the operating polishing spindle on top of a glass lens. The blue flexible tubes are the feeding system for the polishing liquid. The tool is moved over the lens surface along a calculated path using the industrial robot. This tool path usually consists of a grid of discrete points with dedicated velocity in between. Surfaces can be processed by e.g. a meander or a spiral path that covers the whole surface.

Optimizing the feed rate for the tool by using computer con- trolled polishing (CCP) minimizes the process time and enables the correction of surface errors by generating a matched removal profile.

Available polishing machines often require a removal profile from the tool as input. It often is generated on a test part and used for the optimization of the whole surface [1,2]. Another possibility is the generation of the removal profile using a simulation of the polishing process [3]. Typically for every point in the tool path a removal profile is generated and can be used in the optimization.

For rotation symmetric surfaces the processing time is reduced when the surface and tool are rotating. This approach is frequently used in the first polishing run that removes the subsurface damage of the grinding process. With a rotating work piece the relative velocity between tool and surface depends on the position of the tool.

The polishing work being done in time $t$ according to Preston in [4] is calculated after:

$$
w=\mu * A * p * v * t
$$

where $w$ is work $[N * m], \mu$ is friction coefficient , $p$ is pressure $\left[\mathrm{N} / \mathrm{m}^{2}\right] A$ is Area $\left[\mathrm{m}^{2}\right]$, and $v$ is relative velocity $[\mathrm{m} / \mathrm{s}]$.

For a precise prediction of the surface removal and therefore a good polishing result all major process parameters according to the Preston equation above have to be known and controlled. Area, pressure, relative velocity and time can be calculated quite easily. Only the friction coefficient is depending on a lot of process parameters such as chemistry, grain size, pad structure or pressure which are not easily accessible. Its dependency on the relative velocity between tool and surface 


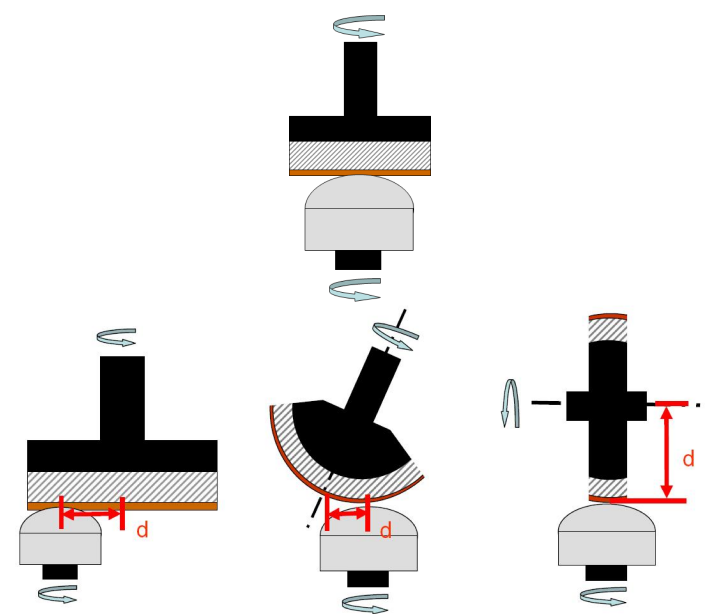

FIG. 2 from top: pole down tool, bottom, left to right: flat offset tool, spherical offset tool, wheel tool

is experimentally shown and verified using a numeric simulation.

\section{POLISHING TOOL}

In Figure 2 a tool setup is shown that is widely used. The polishing tool usually consists of foam that is shown as shaded area. It enables the adaption of the tool to the surface. The polishing pad is shown in brown and is in contact with the work piece and carries the polishing liquid. When the tool is used in pole down mode the rotation axis of the tool is the center of the area of contact. This area of contact is shifted when an offset $d$ is used, as shown in Figure 2.

The offset mode has the advantage of a higher relative velocity compared to the pole down mode as shown in Figure 4. The dotted red line indicates the relative velocity of a pole down tool while tool and surface are rotating with $700 \mathrm{rpm}$. The solid red line represents the relative velocity of an offset tool using the same rotational speed. With an offset $d$ of $30 \mathrm{~mm}$ a substantial increase of the relative velocity can be seen.

According to Preston the relative velocity between surface and tool is linear dependent on the surface removal [4]. The relative velocity between tool and surface is a basic parameter related to rotational speed of tool and surface.

The relation between relative velocity and friction coefficient has a major impact on the local removal rate of the tool and therefore on the global variation of the removal rate.

\section{FRICTION COEFFICIENT}

The dependency of the friction coefficient on the relative velocity is demonstrated by two polishing experiments that are each carried out using an offset of $0 \mathrm{~mm}$ and $30 \mathrm{~mm}$.

The tool is moved over the surface along the green tool path shown in Figure 3 using a given feed rate. The area of contact between tool and surface is marked in blue. The rotational speeds of the tool $\omega_{s}$ and the surface $\omega_{t}$ are controlled and can

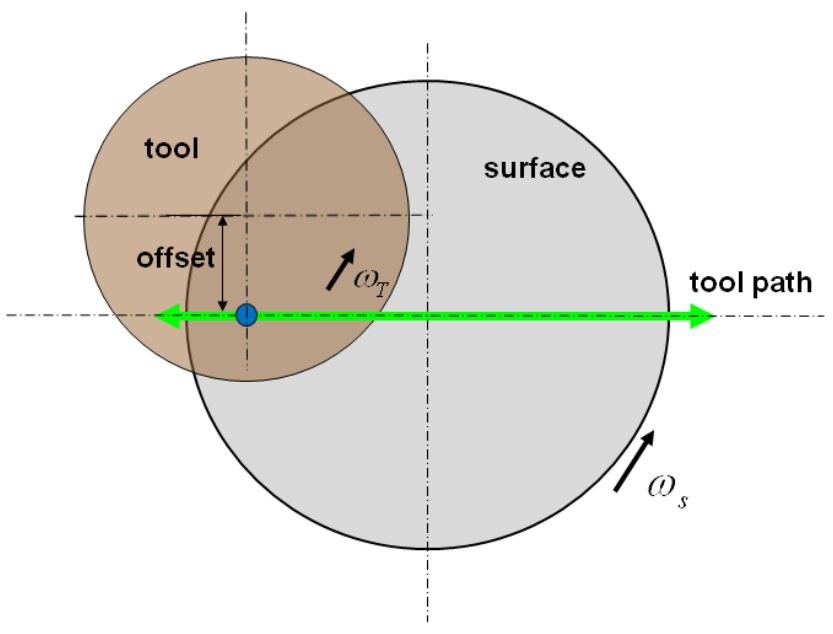

FIG. 3 Offset tool setup for polishing experiment

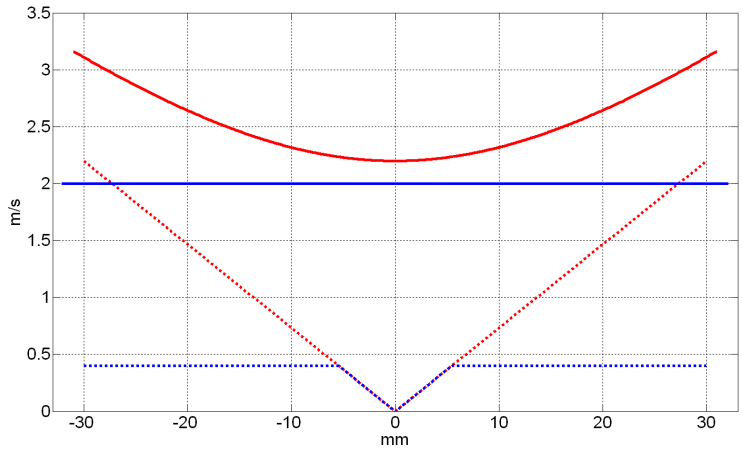

FIG. 4 Absolute value of relative velocities for polishing experiments, solid: offset mode, dotted: pole down mode

be adjusted for each point along the green tool path in Figure 3.

The first experiment uses a constant feed rate and fixed rotational speeds of $700 \mathrm{rpm}$. This generates a varying relative velocity depending on the tool position on the surface. The solid red line in Figure 4 represents the absolute value of the relative velocity in relation to the position on the tool path for the offset mode, the dotted red line for the pole down mode.

The second experiment uses a matched feed rate and rotational speed profiles for tool and surface that generates a constant relative velocity, shown as blue lines in Figure 4 . The relative velocity was constant $2 \mathrm{~m} / \mathrm{s}$ over the whole surface for the offset mode (solid blue line). The pole down mode shown as dotted blue line has a zero point in the centre. For that reason a constant relative velocity could not be achieved over the whole surface. The rotational speeds where kept at $700 \mathrm{rpm}$ for distances below $5.5 \mathrm{~mm}$ around the centre and adjusted otherwise to achieve a constant relative velocity of $0.4 \mathrm{~m} / \mathrm{s}$.

Both polishing experiments where run on a spherical surface, using a normal force $F_{N}$ of $40 \mathrm{~N}$ on the polishing pad. The duration for experiment one was 66 minutes and for experiment two 56 minutes.

In addition to the polishing on the robot a simulation of the polishing wear was run using the model of nonlinear unconnected springs for the tool and discrete time steps 

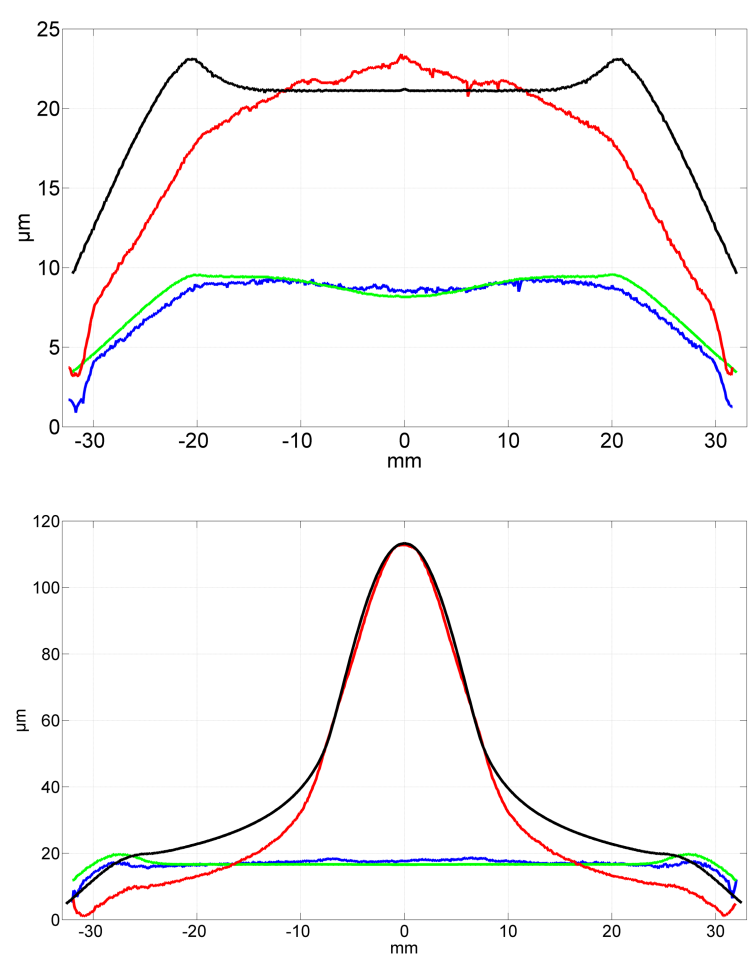

FIC. 5 top: pole down tool, bottom: offset tool

black: Simulation of wear with variable relative velocity, red: polishing of wear with variable relative velocity, green: simulation of wear with fixed relative velocity, blue: wear with fixed relative velocity

for the calculation of the surface wear as described in [3]. The friction coefficient was assumed to be constant for the whole simulation. The other parameters $\omega_{t}, \omega_{s}, F_{N}$ and feed rate where identical to those of the two polishing experiments.

The black and red lines in Figure 5 are the results of simulation and a real polishing run of experiment one (constant rpm) while the green and blue lines are the results of simulation and real polishing run of experiment two (matched rpm).

A decrease of the rotational speeds generates a decrease in the amount of material removed per time. Comparing the black and green lines or the red and the blue lines illustrates that.

More important than that is the difference between the black and the red lines. This indicates that the simulation predicts too much removal when the tool is not in the centre. The comparison of the green and blue lines shows a much better match in the experiment using constant relative velocity.

The parameters of both experiments where kept constant. Only the feed rate profile and the rotation profiles for tool and surface where adapted. This implies that the reason for the difference of the simulation and the polishing wear is connected to the variation of the relative velocity in the first run.

The assumption of a constant friction coefficient that was used in the simulation seems to be the source of the discrepancy seen in this type of polishing.

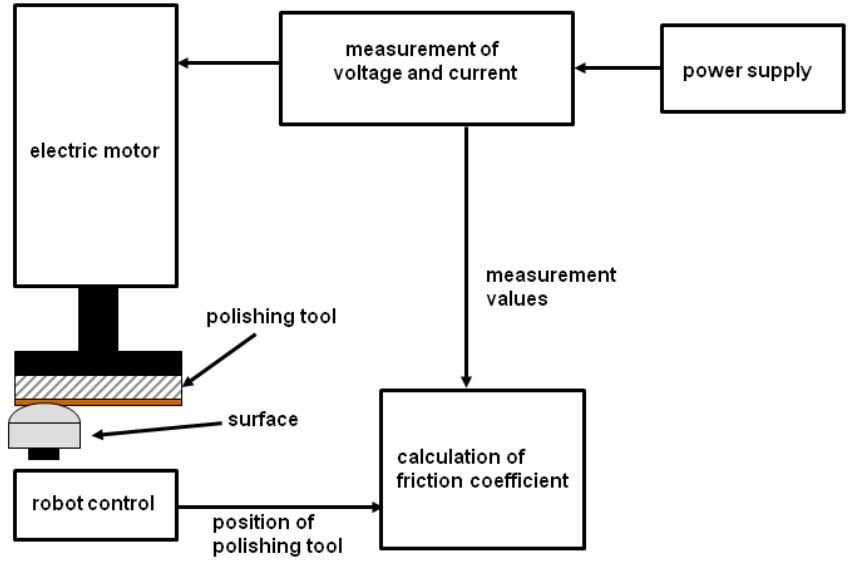

FIC. 6 Setup for measuring the friction coefficient

A constant friction coefficient is only valid if the variation of the relative velocity can be neglected e.g. with fixed work piece. In all other cases the dependency between friction coefficient and relative velocity can have heavy influences e.g. with rotating work pieces using small subaperture tools on the local removal rate.

\section{MEASUREMENT OF THE FRICTION COEFFICIENT}

The measurement principle is derived from the polishing process using an offset tool. It can be implemented permanently in a polishing process used in production. The field of application would range from measurement of the friction coefficient to process monitoring of tool and polishing liquid.

The friction coefficient varies with polishing time [5]-[7] and it also depends on the static and dynamic properties of the materials in use. A fast inline measurement process of the friction coefficient improves the prediction of the removal rate.

Figure 6 shows the measurement principle using the measurement of the effective electric power from the polishing tool as indicator. The voltage and the current of the electric power supply are measured continually and the effective position of the polishing tool on the surface is recorded.

In principle it is also possible to use the power measurement of the motor rotating the lens. The offset variable in Eq. (3) then has to be adapted accordingly.

The tool in Figure 7, shown in brown, is moved along the tool path shown in green over the center of the surface. The area of contact between tool and surface is shown in blue. The tool is chosen in a way that the area of contact is kept very small. Therefore only the center point of the contact zone is considered in the calculation.

The velocity components of tool $v_{x}$ and surface $v_{T}$ then point in the same direction. The resulting relative velocity is received with a linear subtraction of both components. $\omega_{S}$ is the rotation speed for the surface and $\omega_{t}$ for the tool and in this 


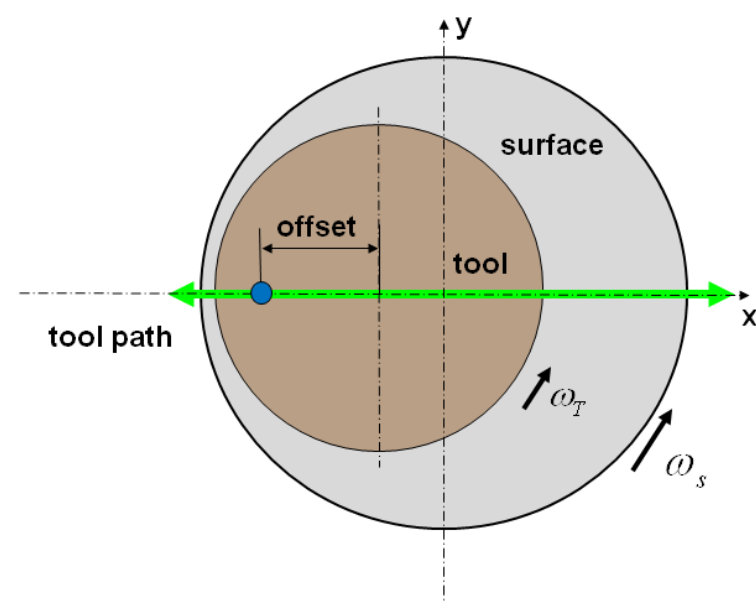

FIC. 7 Usage of polishing tool for measurement of friction coefficient

$$
\begin{gathered}
\text { experiment } \omega_{s}=\omega_{t}=\omega . \\
v_{s}=\omega_{s} \cdot(x+\text { offset }) \\
v_{T}=\omega_{T} \cdot \text { offset } \\
v_{R e l}=v_{s}-v_{t}=\omega \cdot x
\end{gathered}
$$

The relevant forces for the calculation of the friction coefficient $\mu$ between surface and tool are the force of friction $F_{f}$ and the normal force $F_{N}$. The normal force is applied on the polishing pad perpendicular to the surface. It is typically known and can be adjusted in the polishing machine. The friction coefficient $\mu$ then can be defined as

$$
\mu=\frac{F_{f}}{F_{N}}
$$

For the tool a moment of force $\tau$ takes effect

$$
\tau=\text { offset } \cdot F_{f}
$$

The power of the drive of the polishing pad $P$ can be expressed as

$$
P=\tau \cdot \omega_{t}
$$

$\omega_{t}$ is typically known and controlled in the polishing machine.

To calculate the friction coefficient, the effective power of the polishing pad is measured and the moment of force $\tau$ calculated with Eq. (5). The Force of friction $F_{f}$ can be calculated with the known offset between rotation axis and area of contact using Eq. (4). Finally the friction coefficient $\mu$ is calculated using Eq. (3). The position of the tool along the tool path is known so the relative velocity between surface and tool can be calculated using Eq. (2c).

The experiment was conducted on a robot ABB IRB 4400 set up as polishing machine. The normal force $F_{N}$ of $40 \mathrm{~N}$ was applied by an air cylinder. The spherical surface made from BK7 had a radius of curvature of about $88 \mathrm{~mm}$ and a diameter of $64 \mathrm{~mm}$. The tool had a diameter of $100 \mathrm{~mm}$ using an offset of $20 \mathrm{~mm}$. Tool and surface rotated with $700 \mathrm{rpm}$.

The black line in Figure 8 illustrates the resulting friction coefficient (y-axis) in relation to the relative velocity ( $x$-axis). The measurement uncertainty is shown by the grey lines.

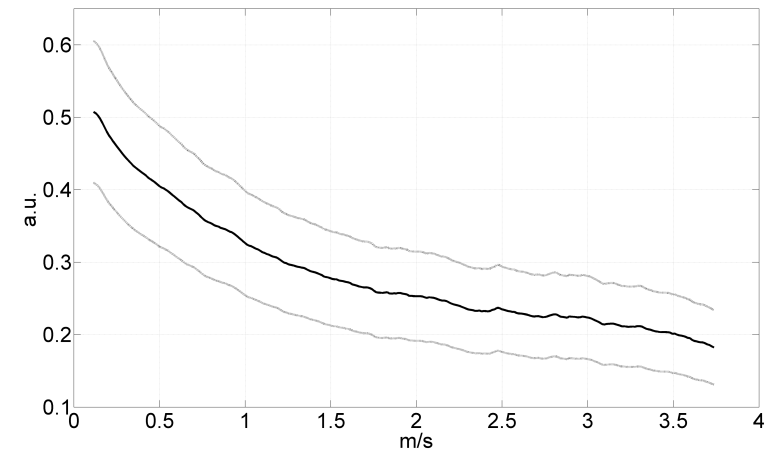

FIG. 8 Calculated friction coefficient vs. relative velocity

The experiment shows that the friction coefficient strongly depends on the relative velocity. It changes with more than a factor of 2 comparing a velocity of $2.5 \mathrm{~m} / \mathrm{s}$ with the measurement data of around $0.1 \mathrm{~m} / \mathrm{s}$.

This has to be taken into account for calculation the local removal rate with the Preston equation. Instead a constant $\mu$ a $\mu(v)$ has to be taken into account.

\section{SUMMARY}

The variation of the friction coefficient has an essential influence on the surface removal of the polishing process. Two experiments with different polishing tools have been presented that show this dependence. In addition a method has been presented that enables the measurement of the friction coefficient. This leads to an enhancement of the polishing process and enables an in situ machine monitoring of the polishing process. The prediction of the local removal in Computer Controlled Polishing improves significant.

\section{References}

[1] S. D. Jacobs, S. A. Arrasmith, I. A. Kozihnova, L. L. Gregg, A. B. Shorey, H. J. Romanofsky, "An overview of magnetorheological finishing (MRF) for precision optics manufacturing," Ceram. Trans. 102, (1999).

[2] W. A. C. M. Messelink, R. Wäger, M. Meeder, T. Wons, K. C. Heiniger, 0. W. Fähnle, "Applying Fluid Jet Polishing for Shape Corrections as well as for pre-polishing Optical Surfaces," in Proceedings of Optifab 2005 (Spie, Rochester, 2005).

[3] A. Kelm, R. Boerret, S. Sinzinger, "Modeling of the polishing process for aspheric optics," SPIE Proc. Ser. 7102, (2008).

[4] F. W. Preston, "The Theory and Design of Plate Class Polishing Machines," J. Soc. Glass Technol. 11, 214-256 (1927).

[5] N. Belkhir, D. Bouzid, V. Herold, "Determination of the Friction Coefficient During Glass Polishing," Tribol. Lett. 33, 55-61 (2009).

[6] Y. Moon, "Investigation of the Relationship between Preston's Coefficient and Friction Coefficient in Chemical Mechanical Polishing (CMP)," http://Ima.berkeley.edu/research/1998/98_Moon_ 1/98_Moon_1.pdf (1998).

[7] S. Yeruva, Particle scale modeling of material removal and surface roughness in chemical mechanical polishing (PhD thesis, University of Florida, 2005). 